\title{
CHARACTERIZATION OF USER-PERCEIVED QUALITY OF SERVICE (QOS) IN MOBILE DEVICES USINg NeTwORK PAIRWISE COMPARISONS
}

\author{
Carlos E. Otero ${ }^{1}$, Ivica Kostanic ${ }^{2}$, Luis D. Otero ${ }^{3}$, and Scott L. Meredith ${ }^{2}$ \\ ${ }^{1}$ Department of Mathematics \& Computer Science, University of Virginia's College at \\ Wise, Wise, VA, USA \\ cotero@virginia.edu \\ ${ }^{2}$ Department of Electrical \& Computer Engineering, Florida Institute of Technology, \\ Melbourne, FL, USA \\ kostanic@fit.edu; smeredit@my.fit.edu \\ ${ }^{3}$ Department of Engineering Systems, Florida Institute of Technology, \\ Melbourne, FL, USA \\ lotero@it.edu
}

\begin{abstract}
This paper presents a user-centric and application-specific QoS assessment methodology for cellular communication networks. Specifically, it uses the Analytic Hierarchy Process (AHP) to evaluate QoS as a multi-criteria decision problem that represents how well cellular networks' data services are perceived given particular sets of application classes and relative to other networks servicing in the same area. As part of the methodology, drive testing is performed to collect objective measurements associated with identified QoS criteria for data services. Once drive testing is performed and data collected, multiple networks are compared to determine the network that provides higher QoS based on users' perception of quality. The selection of the best performing network is based on the output provided by the AHP approach, which is used as unified measurement of the perceived QoS by users on different networks. In order to determine application-specific priorities, the approach presented uses three different application classes, including Emergency, Business, and Personal. For each class, the relative importance of each quality evaluation criteria is adjusted in the AHP procedure to reflect the priorities of the services expected. Through several case studies, the approach is proven successful in providing a way for analyzing user-centric QoS for application-specific scenarios.
\end{abstract}

\section{KEYWORDS}

Quality of Service, Wireless Networks, Cellular Networks, Analytic Hierarchy Process, Performance Evaluation in Cellular Networks

\section{INTRODUCTION}

By 2014, cell phones and other mobile devices will send and receive more data each month than they did in all of 2008 [1]. This fast and constant increase in the cellular market is expected to leave a huge imprint in all facets of human (day to day) life, such as social networking, transportation, healthcare, education, and national security. Unfortunately for network providers, loyalty is a rare (and perhaps non-existent) trait among customers when it comes to choosing/keeping a particular mobile cellular system provider. This means that no matter how much effort, engineering, and money invested by companies into their respective communication technologies, users will ultimately make their decisions based on their perceived quality of service (QoS). Therefore, to remain competitive, network providers will be required to understand and characterize customers' perception of the QoS provided by their respective technologies. 
In today's cellular markets one encounters multiple cellular networks servicing the same geographical area. For example, in mature US markets, there may be as many as eight cellular licenses servicing the area ( 2 in the $850 \mathrm{MHz}$ band and 6 in the $1900 \mathrm{MHz}$ band). Each license may host a different service provider. Furthermore, with recent auctions of $700 \mathrm{MHz}$ spectrum and the rollout of broadband services in $2.5 \mathrm{GHz}$ and $3.65 \mathrm{GHz}$ bands, the number of wireless cellular networks is bound to increase even more. Due to various competitive and historical reasons, deployed networks may be quite different. For example, the networks may have been deployed with different objectives in mind, they may utilize different access technologies or they may be at a different evolutional stage of a given access technology. Furthermore, most of the networks support both circuit switched (CS) and packet switched (PS) communication services. While CS services are dominated by voice, PS services support a plurality of data centric applications. As a result, a comparison between QoS levels offered by different networks becomes a non trivial task. As cellular technology continues to ingrain itself in all aspects of society, network providers must emphasize on delivering high QoS to its end users. Therefore, the assessment of QoS based on user experiences becomes essential.

This paper proposes a quantitative approach for evaluating the perceived QoS of data services in wireless cellular networks. Specifically, the approach uses the Analytic Hierarchy Process (AHP) to evaluate data collected from drive-testing to characterize the perceived QoS of data services from three different networks. This characterization can be used by network providers to evaluate how well their services are being perceived so that they can make corrections or improvements as needed. The development of this methodology provides a unique approach to characterize QoS based on application-specific quality criteria and relative to other networks operating in the same geographical area. The remainder of the paper is organized as follows. Section 2 provides a brief summary of user-centric QoS evaluation methods. Section 3 provides a brief summary of the solution approach, which includes drive-testing and the AHP. Sections 4 and 5 provide detailed explanations of the drive-testing experimental environment and AHP technique. Section 6 presents the results of various case studies. Finally, Section 7 provides summarized conclusions and highlights of the proposed approach.

\section{BACKGROUND WORK}

In [2], the authors present a QoS assessment methodology for cellular communication networks based on the data collected through drive-testing. The work focuses on the end user perception of service quality by providing independent QoS measurement for voice and data services. In their work, the authors discuss QoS assessment for both the circuit switched and packet switched side of the network; however, they fail to provide QoS measurements as function of both voice and data services simultaneously. In [3], the authors stress the importance of network operators evaluating user-perceived QoS of data services in cellular networks. In their work, the authors specify user experience as a key factor in determining the network operator's success; and present a methodology for evaluating quality of the FTP data service in cellular UMTS networks. Their methodology is based on data collected through drive testing and can be easily extended to other cellular data services. However, the proposed methodology concentrates on evaluating end-user experience based only on data services on a single network. Similarly, in [4], the authors present results of evaluation of (user-centric) QoS of background services in an individual UMTS network. In their work, the authors present results for userperceived QoS for email and SMS. However, their approach is bounded to a single UMTS network. In [5], a multi-network methodology for evaluating QoS for both voice and data services is presented. The authors use drive-testing to measure individual QoS criteria for both voice and data services. The criteria used for evaluating QoS in voice services are Accessibility, Retainability, Call Quality, and Voice Quality. For data services, the identified QoS criteria include Connection, FTP Downlink, and FTP Uplink. Once drive testing is performed and the data collected, their approach uses desirability functions to fuse all 
measurements into one unified value that is representative of QoS for a particular application class. In their work, the authors define the following application classes: Emergency, Business, and Personal. For each class, the desirability function parameters are adjusted to reflect the priorities of the (voice and data) services expected. This results in a holistic QoS measurement that considers prioritized voice and data services. The approach provides an improvement to previous work by fusing both voice and data services, and providing a way to customize the evaluation process to specific classes of applications. However, their approach fails to provide evaluation of QoS relative to other networks in the same geographical area. In [6], the authors present a process for comparing Quality of Experience (QoE) in packet-switched networks using the AHP and Grey Relational Analysis. However, by their own admission, their approach, like many other QoE approaches, "rely mostly on user survey and scores from the user, which are too subjective and need much processing time and cost.”[6].

From the reviewed literature, it is evident that there is room for the development of a holistic, user-centric, and application-specific evaluation methodology that provides objective measurements of QoS relative to all other networks available in the same region. This will allow network providers to better assess how well their services are being perceived relative to their competitors.

\section{SOLUTION APPROACH}

To properly evaluate QoS of data services in cellular networks, analysts must follow a methodology that considers user-experiences in specific application scenarios. Furthermore, the evaluation methodology must allow users to compare how well networks perform (relative to competitors) in the same geographical region based on predefined evaluation criteria. The evaluation methodology must also allow users to assign priorities to evaluation criteria to customize the results based on particular classes of applications. The creation of such methodology is achieved as follows. First, drive-testing is performed to collect objective measurements associated with identified QoS criteria for data services. Once drive-testing is completed and the data collected, the AHP technique is used to compare multiple networks and determine the network that provides higher QoS based on users' perception of quality. The output provided by the AHP approach can be used as unified measurement of the perceived QoS by users on different networks. In order to determine application-specific priorities, the approach presented here uses the application classes identified in [5], that is: Emergency, Business, and Personal. For each class, the relative importance of each quality evaluation criteria is adjusted in the AHP procedure to reflect the priorities of the services expected.

\section{DRIVE TESTING}

Drive-testing is performed by placing calls, either voice or data, to a cellular network and recording the data from these calls. The calls are automated by test equipment and the data are geographically referenced by using a GPS unit. A typical setup for the drive testing equipment for PS data measurements is presented in Figure 1. As seen, the setup consists of the in-vehicle equipment and appropriate PS data server. The in-vehicle equipment controls data cards (or other data communicating devices), associated with the cellular networks under test. The PS data server is connected to the cellular networks through the Internet cloud. The connectivity between the PS data server and the Internet is accomplished through high bandwidth dedicated lines. This way the performance of the data service is primarily determined by the performance of the cellular side of the network. The in-vehicle equipment controls data devices and it is programmed to execute a sequence of actions. Depending on what needs to be tested, the sequence may include one or multiple data services. 


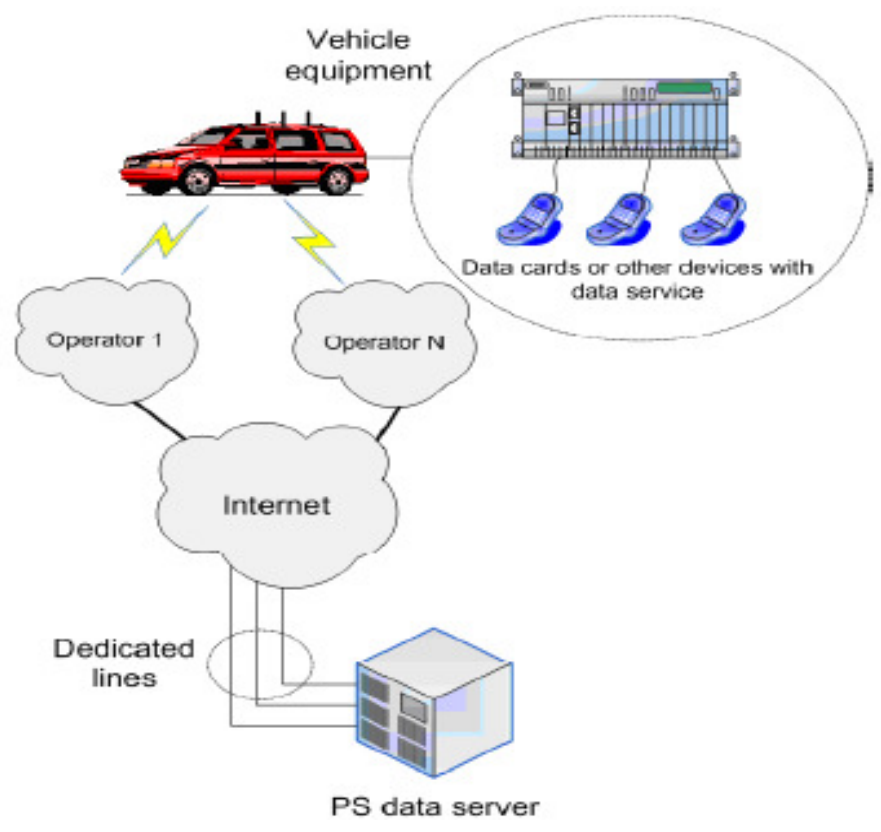

Figure 1. Drive Testing Setup Overview

Each device in the vehicle loops through the same sequence of tasks and relevant measurements are collected, time stamped and geographically referenced. The types of tasks that are included in the test depend on what particular data service needs to be tested. In practice, one would at least test ping, FTP upload, FTP download and HTTP based web browsing [7, 8]. The PS data server needs to be configured properly so that it is able to act as a termination point for all data calls.

\section{Analytic Hierarchy Process}

The AHP is a multi-attribute decision-making method used to facilitate decisions that involve multiple competing goals $[9,10,11]$. It provides a powerful tool that can be used to evaluate different network providers based on multiple QoS Evaluation Criteria (QEC). It starts by transforming the QoS evaluation problem into a structured hierarchy where each QEC is quantified and related to overall goals for evaluating alternative solutions. Typical QEC for data services could include network accessibility, success rates of FTP download, or success rate of FTP uploads. Typical goals for evaluating alternative solutions could include maximizing (or minimizing) all QEC identified. Evaluation goals are customized based on specific application requirements. For example, in the Emergency class of applications (EMTs, police officers, etc.), users will come to rely heavily on location-based services, where connection and downlink data services are of most importance. Other examples include the business and personal class of applications. In all cases, AHP can be used to quantify goals, prioritize them, and include them in the evaluation methodology. A generic AHP hierarchy for the QoS evaluation process is presented in Figure 2.

The second and third levels of the AHP hierarchy vary according to the networks available and the QECs selected for evaluating the networks. As seen, the second level can be extended to include other QECs, such as increased security, maintenance, voice services, etc. The third level consists of the networks servicing the geographical region being evaluated. To describe the methodology, this paper considers the following three networks: CDMA, GSM and UMTS. 


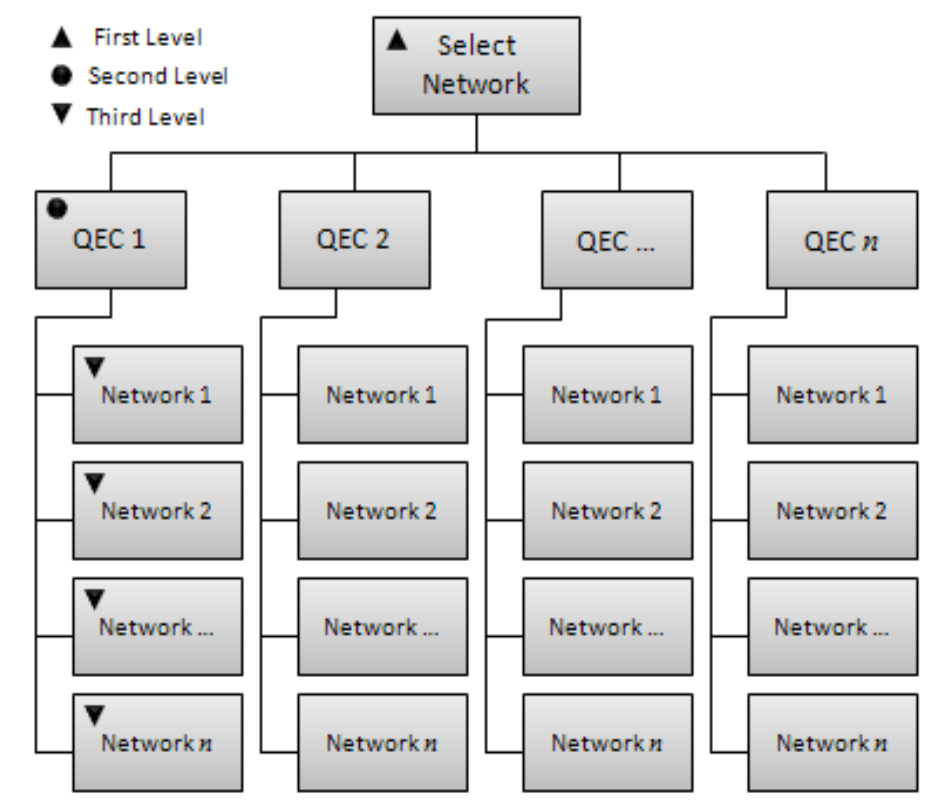

Figure 2. AHP Hierarchy for QoS Evaluation

In other scenarios, there could be $n$ networks, each providing different measurements for each QEC identified. Once the hierarchy is built, and relevant QEC measurements taken for each network, a common scale is created to rank each network. That is, for each comparison made during the AHP, a common pair-wise comparison scale is used to determine how preferred one option is from another. This allows standardization in all comparisons made during the AHP process. Table 1 presents the pairwise comparison scale created for the QoS evaluation problem.

Table 1. Pairwise Comparison Scale.

\begin{tabular}{|c|l|}
\hline Scale $(\boldsymbol{w})$ & Description \\
\hline 1 & Equally Preferred \\
\hline 2 & Equally to Moderately Preferred \\
\hline 3 & Moderately Preferred \\
\hline 4 & Moderately to Strongly Preferred \\
\hline 5 & Strongly Preferred \\
\hline
\end{tabular}

QoS evaluators establish preferences between different networks using the pairwise comparison scale and pairwise comparison matrices [11]. There are two types of pairwise comparison matrices in AHP: the Network vs. Network matrices, and the QEC vs. QEC matrix. The Network vs. Network pairwise comparison matrices are matrices where each element $a_{i j}$ represents how much more desirable the network at row $i$ is than the network at column $i$, in terms of a pre-defined QEC (e.g., connectivity, downlink, uplink). Using the results from drivetesting, QoS evaluators are equipped with the knowledge necessary to make these assignments. The format of the Network vs. Network matrices is presented in (1), where $A_{z}$ is the pairwise comparison matrix for QEC $z$ (i.e., $\mathrm{z} \in\{$ connectivity, downlink efficiency, uplink efficiency $\}$ ) and $N_{x}$ represents network $x$. 


$$
A_{z}=\begin{array}{cccc}
N_{1} & N_{2} & \cdots & N_{n} \\
N_{1} & N_{2} \\
\vdots & N_{n}
\end{array}\left[\begin{array}{cccc}
w_{1} / w_{1} & w_{1} / w_{2} & \cdots & w_{1} / w_{n} \\
w_{2} / w_{1} & w_{2} / w_{2} & \cdots & w_{2} / w_{n} \\
\vdots & \vdots & \ddots & \vdots \\
w_{n} / w_{1} & w_{n} / w_{2} & \cdots & w_{n} / w_{n}
\end{array}\right]
$$

From each $A_{z}$ matrix, a weight vector $\mathrm{W}$ is computed to determine the relative importance of each network in the pairwise comparison matrix. That is, assuming weight vector $W=\left[\begin{array}{llll}w_{1} & w_{2} & \cdots & w_{n}\end{array}\right]$, the value of $w_{i}$ represents the relative importance of network $i$ of the associated pairwise comparison matrix based on QEC $z$. The weight vectors are used to make the final decision. To compute the weight vectors, the pairwise comparison matrix $A_{z}$ is normalized using (2),

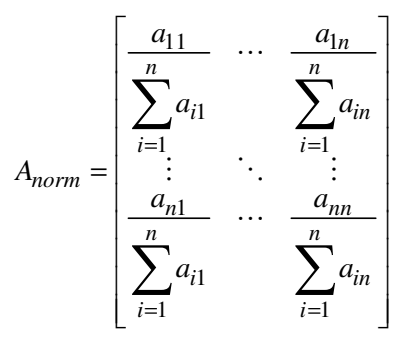

where $a_{i j}$ represents the $a^{\text {th }}$ element at row $i$ and column $j$ of the respective Network vs. Network comparison matrix. Once in normalized form, the weight vector associated with $A_{\text {norm }}$ is computed with (3).

$$
W=\left[w_{1}=\frac{\sum_{j=1}^{n} a_{1 j}}{n} \quad w_{2}=\frac{\sum_{j=1}^{n} a_{2 j}}{n} \quad \cdots \quad w_{n}=\frac{\sum_{j=1}^{n} a_{n j}}{n}\right]
$$

The QEC vs. QEC pairwise comparison matrix is a $n x n$ matrix where each location $a_{i j}$ represents how much more important the QEC (e.g., connectivity, downlink, uplink, etc.) at row $i$ is than the QEC at column $j$. The importance of each QEC is configured depending on the class of application (e.g., Emergency, Business, or Personal) used in the evaluation process. The format of the QEC vs. QEC matrix is presented in (4), where $w_{i}$ is the weight given to QEC $i$.

$$
A=\begin{gathered}
Q_{1} \\
Q_{1} \\
Q_{2} \\
\vdots \\
Q_{n}
\end{gathered}\left[\begin{array}{cccc}
w_{1} / w_{1} & w_{1} / w_{2} & \cdots & w_{1} / w_{n} \\
w_{2} / w_{1} & w_{2} / w_{2} & \cdots & w_{2} / w_{n} \\
\vdots & \vdots & \ddots & \vdots \\
w_{n} / w_{1} & w_{n} / w_{2} & \cdots & w_{n} / w_{n}
\end{array}\right]
$$

Once the QEC vs. QEC matrix is created, it is normalized and the weight vector is computed using the same procedure as in the Network vs. Network matrices. Once all weight vectors in the QoS evaluation problem have been computed, they are used to determine the network that 
provides the best QoS. For example, assuming a QoS evaluation problem with $x$ number of QEC and $y$ number of networks, the AHP provides $y+l$ weight vectors; one $\left(W_{A}\right)$ associated with the QEC vs. QEC pair-wise comparison matrix, and the rest $W_{i}$ associated with each Network vs. Network matrix $i$, as illustrated in Figure 3.

$$
\begin{gathered}
w_{1}^{T} w_{2}^{T} w_{\ldots}^{T} \quad w_{y-1}^{T} \\
{\left[\begin{array}{c}
w_{1} \\
w_{2} \\
\vdots \\
w_{n}
\end{array}\right]\left[\begin{array}{c}
w_{1} \\
w_{2} \\
\vdots \\
w_{n}
\end{array}\right]\left[\begin{array}{c}
w_{1} \\
w_{2} \\
\vdots \\
w_{n}
\end{array}\right]\left[\begin{array}{c}
w_{1} \\
w_{2} \\
\vdots \\
w_{n}
\end{array}\right] \quad\left[\begin{array}{c}
w_{1} \\
w_{2} \\
\vdots \\
w_{n}
\end{array}\right]}
\end{gathered}
$$

Figure 3. AHP Weight Vectors

To compute the relative preference for network $i$, we let $W=W_{i}, W A=W_{A}$, and define $S_{i}$ as the overall score for network $i$, then,

$$
S_{i}=\sum_{k=1}^{n} W_{k}\left(W A_{k}\right)
$$

where $k$ represents the $k^{\text {th }}$ element of vectors $W$ and $W A$. Once overall scores are computed for all networks, the highest score is identified as the network providing the best QoS, followed by the second highest score, and so on. This prioritized list helps determine the best perceived QoS for a particular class of applications among different network providers.

\section{Case Study}

This section presents results of a QoS evaluation case study using the proposed approach. The case study evaluates QoS of data services for the following three cellular networks: CDMA, GSM, and UMTS. The types of QEC depend on the particular data services being evaluated. For this case study, the identified QEC for data services include Connection (CO), FTP Downlink (DL), and FTP Uplink (UL). CO is a measure of the network accessibility; that is, the ratio between the number of successfully established connections and the number of attempted connections. DL and UL are measurements of the probability that a started FTP download or upload terminates properly with its completion. The file sizes used for evaluating DL and UL were $3 \mathrm{MB}$ and $100 \mathrm{~KB}$ respectively. For details of the drive-testing environment and setup, readers can refer to [2]. The success rates for CO, DL, and UL are presented in Table 2.

Table 2. QoS Criteria Assessment Matrix for Case Study 1

\begin{tabular}{|l|c|c|c|}
\hline \multirow{2}{*}{ Network } & \multicolumn{3}{|c|}{ Data Services Success Rate (\%) } \\
\cline { 2 - 4 } & Connectivity & Downlink & Uplink \\
\hline CDMA & 89.00 & 70.00 & 65.00 \\
\hline GSM & 74.00 & 55.00 & 75.00 \\
\hline UMTS & 83.00 & 88.00 & 94.00 \\
\hline
\end{tabular}

To evaluate the data services QoS provided by the networks, pairwise comparison of each network in terms of each individual QEC is performed. Each network is compared using the comparison scale specified in Table 1. Results are presented below. 
Table 3. Network vs. Network Comparison Matrix, Normalized Matrix, and Weight Vector for Connectivity

\begin{tabular}{|l|c|c|c|}
\cline { 2 - 4 } \multicolumn{1}{c|}{ Connectivity } & CDMA & GSM & UMTS \\
\hline CDMA & 1.00 & 4.00 & 3.00 \\
\hline GSM & 0.25 & 1.00 & 0.33 \\
\hline UMTS & 0.33 & 3.00 & 1.00 \\
\hline Total & 1.58 & 8.00 & 4.33 \\
\hline
\end{tabular}

\begin{tabular}{|l|c|c|c|}
\cline { 2 - 4 } \multicolumn{1}{c|}{ Connectivity } & CDMA & GSM & UMTS \\
\hline CDMA & 0.63 & 0.50 & 0.69 \\
\hline GSM & 0.16 & 0.13 & 0.08 \\
\hline UMTS & 0.21 & 0.38 & 0.23 \\
\hline
\end{tabular}

\begin{tabular}{|l|c|c|c|}
\cline { 2 - 4 } Connectivity & CDMA & GSM & UMTS \\
\hline Weight & 0.61 & 0.12 & 0.27 \\
\hline
\end{tabular}

Table 4. Network vs. Network Comparison Matrix, Normalized Matrix, and Weight Vector for Downlink

\begin{tabular}{|l|c|c|c|}
\cline { 2 - 4 } \multicolumn{1}{l|}{ Downlink } & CDMA & GSM & UMTS \\
\hline CDMA & 1.00 & 4.00 & 0.25 \\
\hline GSM & 0.25 & 1.00 & 0.20 \\
\hline UMTS & 4.00 & 5.00 & 1.00 \\
\hline Total & 5.25 & 10.00 & 1.45 \\
\hline
\end{tabular}

\begin{tabular}{|l|c|c|c|}
\cline { 2 - 4 } \multicolumn{1}{c|}{ Downlink } & CDMA & GSM & UMTS \\
\hline CDMA & 0.19 & 0.40 & 0.17 \\
\hline GSM & 0.05 & 0.10 & 0.14 \\
\hline UMTS & 0.76 & 0.50 & 0.69 \\
\hline
\end{tabular}

\begin{tabular}{|l|c|c|c|}
\cline { 2 - 4 } \multicolumn{1}{l|}{ Downlink } & CDMA & GSM & UMTS \\
\hline Weight & 0.25 & 0.10 & 0.65 \\
\hline
\end{tabular}

Table 5. Network vs. Network Comparison Matrix, Normalized Matrix, and Weight Vector for Uplink

\begin{tabular}{|l|c|c|c|}
\cline { 2 - 4 } \multicolumn{1}{c|}{ Uplink } & CDMA & GSM & UMTS \\
\hline CDMA & 1.00 & 0.33 & 0.20 \\
\hline GSM & 3.00 & 1.00 & 0.25 \\
\hline UMTS & 5.00 & 4.00 & 1.00 \\
\hline Total & 9.00 & 5.33 & 1.45 \\
\hline
\end{tabular}

\begin{tabular}{|l|c|c|c|}
\cline { 2 - 4 } \multicolumn{1}{c|}{ Uplink } & CDMA & GSM & UMTS \\
\hline CDMA & 0.11 & 0.06 & 0.14 \\
\hline GSM & 0.33 & 0.19 & 0.17 \\
\hline UMTS & 0.56 & 0.75 & 0.69 \\
\hline
\end{tabular}

\begin{tabular}{|l|c|c|c|}
\cline { 2 - 4 } \multicolumn{1}{l|}{ Uplink } & CDMA & GSM & UMTS \\
\hline Weight & 0.10 & 0.23 & 0.67 \\
\hline
\end{tabular}


Using the pairwise comparison matrices of all networks based on each QEC, the AHP approach can now be used to compute a measurement of QoS for all three classes of applications identified in [5]; that is, Emergency, Business, and Personal. The following sections show the effects that each of these applications have on overall QoS evaluation. Comparisons of QEC vs. QEC are made to properly reflect the relative importance of each QEC in the three types of applications.

\subsection{Emergency Class}

To show the effects that particular classes of applications have on overall QoS evaluation, the QEC vs. QEC comparisons are made to properly reflect the relative importance of each QEC in emergency applications (EMTs, police officers, etc). In this class of applications, users will come to rely heavily on location-based services, where connection and downlink data services are of most importance. The QEC vs. QEC comparison matrix, normalized matrix, and weight vector are presented in Table 6.

Table 6. QEC vs. QEC Matrix, Normalized Matrix, and Weight Vector for Emergency Class

\begin{tabular}{|l|c|c|c|}
\cline { 2 - 4 } \multicolumn{1}{l|}{ QEC vs. QEC } & Connectivity & Downlink & Uplink \\
\hline Connectivity & 1 & 4 & 5 \\
\hline Downlink & 0.25 & 1 & 4 \\
\hline Uplink & 0.2 & 0.25 & 1 \\
\hline Total & 1.45 & 5.25 & 10 \\
\hline
\end{tabular}

\begin{tabular}{|l|c|c|c|}
\cline { 2 - 4 } \multicolumn{1}{l|}{ QEC vs. QEC } & Connectivity & Downlink & Uplink \\
\hline Connectivity & 0.69 & 0.76 & 0.50 \\
\hline Downlink & 0.17 & 0.19 & 0.40 \\
\hline Uplink & 0.14 & 0.05 & 0.10 \\
\hline
\end{tabular}

Weight

\begin{tabular}{|c|c|c|}
\hline Connectivity & Downlink & Uplink \\
\hline 0.65 & 0.25 & 0.10 \\
\hline
\end{tabular}

Finally, using (5), the results of Tables $3-6$ are combined to provide the final QoS measurement for data services based on the Emergency class of applications. The final perceived QoS measurement is presented in Table 7.

Table 7. Network QoS for Data Services in the Emergency Class

\begin{tabular}{|l|c|}
\cline { 2 - 2 } \multicolumn{1}{l|}{ Network } & QoS \\
\hline CDMA & $47.00 \%$ \\
\hline GSM & $12.42 \%$ \\
\hline UMTS & $40.57 \%$ \\
\hline
\end{tabular}

\subsection{Business Class}

For Business applications, the ability to connect and uplink a file may be of higher importance than the downlink capability. Therefore, for this case application, the QEC vs. QEC comparison matrix is configured to reflect this assumption, as seen in Table 8. The QEC vs. QEC comparison matrix, normalized matrix and weight vector are presented in Table 8 and the results presented in Table 9. 
Table 8. QEC vs. QEC Comparison Matrix, Normalized Matrix, and Weight Vector for Business Class

\begin{tabular}{|l|c|c|c|}
\cline { 2 - 4 } \multicolumn{1}{c|}{ QEC vs. QEC } & Connectivity & Downlink & Uplink \\
\hline Connectivity & 1.00 & 3.00 & 0.50 \\
\hline Downlink & 0.33 & 1.00 & 0.20 \\
\hline Uplink & 2.00 & 5.00 & 1.00 \\
\hline Total & 3.33 & 9.00 & 1.70 \\
\hline
\end{tabular}

\begin{tabular}{|l|c|c|c|}
\cline { 2 - 4 } \multicolumn{1}{l|}{ QEC vs. QEC } & Connectivity & Downlink & Uplink \\
\hline Connectivity & 0.30 & 0.33 & 0.29 \\
\hline Downlink & 0.10 & 0.11 & 0.12 \\
\hline Uplink & 0.60 & 0.56 & 0.59 \\
\hline
\end{tabular}

Weight

\begin{tabular}{|c|c|c|}
\hline Connectivity & Downlink & Uplink \\
\hline 0.31 & 0.11 & 0.58 \\
\hline
\end{tabular}

Table 9. Network QoS for Data Services in the Business Class

\begin{tabular}{|l|c|}
\cline { 2 - 2 } \multicolumn{1}{l|}{ Network } & QoS \\
\hline CDMA & $27.62 \%$ \\
\hline GSM & $18.18 \%$ \\
\hline UMTS & $54.20 \%$ \\
\hline
\end{tabular}

As seen, when uplink is equally to moderately preferred to connectivity; connectivity moderately preferred to downlink; and uplink strongly preferred to downlink, the results of the perceived QoS change to identify the UMTS network as the best performer, followed by CDMA, and GSM. In similar fashion, the QEC vs. QEC parameters can be adjusted for applications in the personal class of mobile applications to appropriately evaluate QoS.

\subsection{Personal Class}

For Personal applications, the ability to download information, such as web pages, music, and pictures are commonly expected among users of the Personal class of applications. Users in this class tend to accept the fact that minimal network flaws related to establishing connections is an inherently and unavoidable part of network services. Therefore, users of the Personal class of applications may put up with not being able to connect to the network at times; however, when a connection is made, they may mandate the ability to download data contents using the network. This can be reflected by configuring the QEC vs QEC matrix to reflect Downlink having the higher priority, followed by Connectivity and Uplink. The QEC vs. QEC comparison matrix, normalized matrix and weight vector are presented in Table 10 and the results presented in Table 11.

Table 10. QEC vs. QEC Comparison Matrix, Normalized Matrix, and Weight Vector for Personal Class

\begin{tabular}{|c|c|c|c|}
\cline { 2 - 4 } \multicolumn{1}{c|}{$\begin{array}{c}\text { Factor vs. } \\
\text { Factor }\end{array}$} & Connectivity & Downlink & Uplink \\
\hline Connectivity & 1.00 & 0.50 & 4.00 \\
\hline Downlink & 2.00 & 1.00 & 5.00 \\
\hline Uplink & 0.25 & 0.20 & 1.00 \\
\hline Total & 3.25 & 1.70 & 10.00 \\
\hline
\end{tabular}


International Journal of Wireless \& Mobile Networks ( IJWMN ), Vol.2, No.3, August 2010

\begin{tabular}{|c|c|c|c|}
\hline $\begin{array}{l}\text { Factor vs. } \\
\text { Factor }\end{array}$ & Connectivity & Downlink & Uplink \\
\hline Connectivity & 0.31 & 0.29 & 0.40 \\
\hline Downlink & 0.62 & 0.59 & 0.50 \\
\hline Uplink & 0.08 & 0.12 & 0.10 \\
\hline \multirow[b]{2}{*}{ Weight } & Connectivity & Downlink & Uplink \\
\hline & 0.33 & 0.57 & 0.10 \\
\hline
\end{tabular}

Table 11. Network QoS for Data Services in the Personal Class

\begin{tabular}{|c|c|}
\cline { 2 - 2 } \multicolumn{1}{c|}{ Network } & QoS \\
\hline CDMA & $35.76 \%$ \\
\hline GSM & $11.68 \%$ \\
\hline UMTS & $52.56 \%$ \\
\hline
\end{tabular}

\section{CONCLUSION}

The research presented in this paper develops an approach for evaluating application-specific and user-perceived QoS in cellular networks based on data services. Specifically, it presents a technology-agnostic methodology that uses AHP to create a unified measurement that represents how well cellular networks operate for a particular set of application classes and relative to other networks servicing the same area. Through several case studies, the approach is proven successful in providing a way for analyzing user-centric QoS for application-specific usage.

There are several important contributions from this research. First, the approach is simple and readily available for implementation using a simple spreadsheet. This can promote usage in practical scenarios, where highly complex methodologies for QoS evaluation are impractical. Second, the approach fuses unlimited QoS quality evaluation criteria to provide a holistic view of the experienced QoS. This allows the approach to be easily extended to include additional data (or voice) quality criteria not considered in this research. Third, by using data from drivetesting, the approach provides objective measures of QoS, which improves previous approaches using subjective data from surveys. Finally, the approach provides a mechanism to evaluate QoS based on application-specific scenarios. By modifying the parameters of the QEC vs. QEC comparison matrix, QoS can be evaluated by taking consideration of prioritized services that are necessary for different classes of applications. Overall, the approach presented in this research proved to be a feasible technique for efficiently evaluating the perceived QoS in wireless cellular networks.

\section{ACKNOWLEDGEMENT}

The authors would like to express a sincere appreciation to QualiTest Technologies, Inc. for support of the research presented in this paper. Also, the authors would like to thank the reviewers whose constructive critique greatly improved the quality of the paper.

\section{REFERENCES}

[1] Cherry, S., "Forecast for Cloud Computing: Up, Up, and Away," IEEE Spectrum Magazine, pp. 68, October, 2009. 
[2] Kostanic, I., Mijatovic, N., Vest, S.D., "Measurement Based QoS Comparison of Cellular Communication Networks," In proceedings of 2009 IEEE International CQR Workshop, Naples, FL, May 2009.

[3] Weissberger, I., Mijatovic, N., Kostanic, I., "Evaluating FTP QoS in a UMTS Networks," In proceedings of 2009 International Conference on Wireless Networks, Las Vegas, NV, July 2009.

[4] Weissberger, I., Kostanic, I., Otero, C.E., "Background Service QoS in a UMTS Network," Proceedings of IEEE SoutheastCon 2010, pp. 230 - 233,, 2010.

[5] Otero, C.E., Kostanic, I., Mijatovic, N., Weissberger, I., "A Holistic Approach for Evaluating Quality of Service (QoS) in Wireless Cellular Networks," Proceedings of 2010 International Conference on Wireless Networks (ICWN), Vol 2., pp. 483 - 487, 2010.

[6] Markaki, O., Charilas, D., Kikitopoulos, D., "Enhancing Quality of Experience in Next Generation Networks Through Network Selection Mechanism," $18^{\text {th }}$ Annual IEEE International Symposium on Personal, Indoor and Mobile Radio Communications, 2007.

[7] Weissberger, I., Kostanic, I., Mijatovic, N., Otero, C.E., "Characterization of HTTP QoS in a UMTS Network," Proceedings of 2010 International Conference on Wireless Networks (ICWN), Vol. 1, pp. 24 - 29, 2010.

[8] Weissberger, I., Kostanic, I., Mijatovic, N., Otero, C.E., "Evaluation of HTTP QoS in a UMTS Network," Proceedings of $3^{\text {rd }}$ International Workshop on Wireless Multimedia Computing and Security Services, June, 2010.

[9] de Steiguer, J.E., Duberstein, J., Lopes, V., The Analytic Hierarchy Process as a Means for Integrated Watershed Management, http://www.tucson.ars.ag.gov/icrw/Proceedings/Steiguer.pdf, Retrieved on August 9, 2008

[10] Winston, W.L., "Operations Research: Applications and Algorithms," Duxbury Press, July, 2003.

[11] Otero, C.E., Kostanic, I., Otero, L.D., “A Decision-making Methodology for Stochastic Deployments of Wireless Sensor Networks," Proceedings of $11^{\text {th }}$ International Conference on Computer Modeling and Simulation," pp. 140 - 145, 2009.

[12] ITU-T: Recommendation G.107 - The E-model, a computational model for use in transmission planning, International Telecommunication Union, 2005.

[13] ITU-T: Recommendation P.800 (08/96) Methods for subjective determination of transmission quality, International Telecommunication Union, 1996.

\section{Authors}

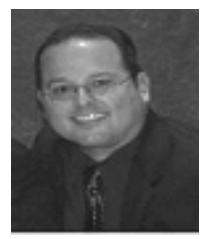

Dr. Carlos E. Otero was born in 1977 in Bayamon, Puerto Rico. He received his B.S. in computer science, M.S. in software engineering, M.S. in systems engineering, and Ph.D. in computer engineering from Florida Institute of Technology, in Melbourne, FL. His primary research interests include performance evaluation and optimization of systems and processes in a wide variety of domains (including wireless systems, software engineering, and systems engineering).

$\mathrm{He}$ is currently Assistant Professor in the department of Mathematics and Computer Science at the University of Virginia's College at Wise, Wise, VA. Previously, he was adjunct professor in the department of Electrical \& Computer Engineering at Florida Institute of Technology. He has over 10 years of industry experience in satellite communications systems, command \& control systems, wireless security systems, and unmanned aerial vehicle systems. 
Dr. Otero is a professional member of the ACM and senior member of the IEEE.

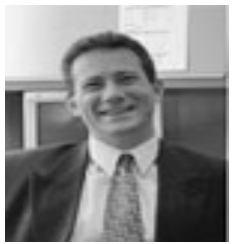

Dr. Ivica Kostanic was born in 1968 in Belgrade, Yugoslavia. He obtained BSEE, MSEE and $\mathrm{PhD}$ from Belgrade University, Florida Institute of Technology and University of Central Florida respectively. His principal research interests include various topics in radio communication, cellular systems and wireless sensor networks. Currently, he is faculty member in the Electrical and Computer Engineering department at Florida Institute of Technology where he teaches courses related to wireless communication, personal communication and microwave circuit design. $\mathrm{He}$ is the technical director of Wireless Center of Excellence (WiCE) which is a group at Florida Tech dedicated to promoting research in wireless communication and computing technologies.

Dr. Kostanic is a member of the IEEE Communication Society.

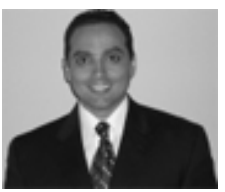

Dr. Luis Daniel Otero was born in 1975 in Bayamon, Puerto Rico. He received his B.S. in Civil Engineering, M.S. in Computer Information Systems, and M.S. in Engineering Management from Florida Institute of Technology, Melbourne, FL. He received his Ph.D. in Industrial and Management Systems Engineering from the University of South Florida, Tampa, FL. His research areas include simulation modeling, decision analysis, project engineering, and systems engineering.

$\mathrm{He}$ is an Assistant Professor of Engineering Systems at Florida Institute of Technology. He has over 8 years of experience as a software engineer for Harris Corporation (Government Communications Systems Division) and Northrop Grumman Corporation (Aerospace Systems). 